\title{
A Discrete Complex Compliance Spectra Model of the Nonlinear Viscoelastic Creep and Recovery of Microcellular Polymers
}

\author{
WILLIAM D. ARMSTRONG, ${ }^{1}$ VIPIN KUMAR ${ }^{2}$ \\ ${ }^{1}$ State University of New York at Binghamton, Thomas J. Watson School of Engineering and Applied Science, \\ Department of Mechanical Engineering, P.O. Box 6000, Binghamton, New York 13902-6000 \\ ${ }^{2}$ University of Washington, Department of Mechanical Engineering, Box 352600, Seattle, Washington 98195
}

Received 12 July 1999; revised 6 December 1999; accepted 7 December 1999

\begin{abstract}
The present work reports a discrete stress-dependent, complex compliance spectra method that may be used to predict the mechanical response of nonlinear viscoelastic polymers during creep and recovery processes. The method is based on the observation that the real and imaginary parts of a discrete complex compliance frequency spectra obtained from creep and recovery measurements are smooth, easily fit functions of stress. The new method is applied to a set of microcellular polycarbonate materials with differing relative density. The nonlinear viscoelastic characteristics of a microcellular polycarbonate material system are very sensitive to relative density and therefore, this material system is a particularly difficult modeling challenge. However, the present model was able to exhibit excellent quantitative agreement with the basis creep and recovery measurements at all experimental stress levels for each of the experimental relative density material types. (C) 2000 John Wiley \& Sons, Inc. J Polym Sci B: Polym Phys 38: 691-697, 2000
\end{abstract}

Keywords: nonlinear viscoelasticity; microcellular polymers; creep and recovery; discrete Fourier series method

\section{INTRODUCTION}

Microcellular foams are generally closed-cell foams with average cell size of order 10 micrometers. ${ }^{1}$ Such foams are characterized by a high density of cells, usually exceeding 100 million cells per cubic centimeter. The high cell density makes it possible to achieve significant reductions in density of the polymer while keeping the average cell size small. Such foams have been created in polycarbonate using carbon dioxide as the gas for bubble nucleation. ${ }^{2}$ The polycarbonate-carbon dioxide system has been a model microcellular system, and a number of investigations have

Correspondence to: W. D. Armstrong (E-mail: wda@ binghamton.edu)

Journal of Polymer Science: Part B: Polymer Physics, Vol. 38, 691-697 (2000)

(C) 2000 John Wiley \& Sons, Inc. focused on the properties of this system. For example, tensile, fatigue, and fracture toughness studies have uncovered some unique properties of these novel foams. ${ }^{3-5}$ A key advantage offered by microcellular foams is the potential to reduce the amount of material needed by replacing the solid polymer with a microcellular polymer of reduced density. As polycarbonate is used in many loadbearing applications, a knowledge of the creep and recovery behavior of microcellular polycarbonate is of special interest.

Wing et al. ${ }^{6}$ presented a model for the creep and recovery response of microcellular polycarbonate based on Schapery's theory of nonlinear viscoelasticity. This article re-examines the experimental measurements of Wing ${ }^{7}$ with a new stress-dependent, discrete dynamic compliance spectra model. The new model requires a set of 
creep and recovery curves measured under a set of constant creep stresses as input, and then predicts the strain under general conditions of time and stress as output. Our interest is to determine whether the new model is able to accurately predict and in some sense explain the nonlinear creep and recovery behavior of microcellular polycarbonate over a broad stress-relative density range.

\section{EXPERIMENTAL MEASUREMENTS}

An experimental investigation of the time-dependent response of microcellular polycarbonate foams was conducted by Wing ${ }^{7}$ on foams of relative densities (density of foam divided by the density of polycarbonate) of $1.00,0.95$, and 0.81 . The foams were produced by first saturating polycarbonate sheets with $\mathrm{CO}_{2}$ gas under a pressure of $5.52 \mathrm{MPa}$ for $60 \mathrm{~h}$ and then releasing the pressure and rapidly heating the sample to $60{ }^{\circ} \mathrm{C}$ (relative density equal to 0.95 ) or $80{ }^{\circ} \mathrm{C}$ (relative density equal to 0.81 ) to create a uniform dispersion of very fine bubbles. In the relative density equal to 0.95 material, the average bubble size was 1.65 $\mu \mathrm{m}$, with approximately $6.510 \mathrm{e} 9$ bubbles per cubic centimeter. In the relative density equal to 0.81 material, the average bubble size was 3.14 $\mu \mathrm{m}$, with approximately $7.810 \mathrm{e} 9$ bubbles per cubic centimeter. The foaming temperature was then held constant for $30 \mathrm{~s}$ after which the microcellular polymer was quenched in room temperature water to terminate the foaming process. The relative density equal to 1.0 case was a solid polycarbonate, which had been saturated with $\mathrm{CO}_{2}$ gas in the same way as the lower relative density cases and then desorbed for at least 60 days. During creep and recovery testing, the specimens were subjected to various levels of constant tension for $8 \mathrm{~h}$, after which the load was removed and the recovery strain monitored for an additional $2 \mathrm{~h}$ in the relative density equal to 0.95 and 0.81 cases; meanwhile, the recovery strain was monitored for an additional $8 \mathrm{~h}$ in the relative density equal to 1.00 case.

Figure 1 shows the experimental creep and recovery measurements. The experimental data is plotted with the same strain axis scale to highlight the very much higher creep strain accumulation occurring in the relative density equal to 0.95 and 0.81 cases as compared to the relative density equal to 1.00 case. The absolute amount of strain recovery increases with increasing applied stress in each material. However, the recov-
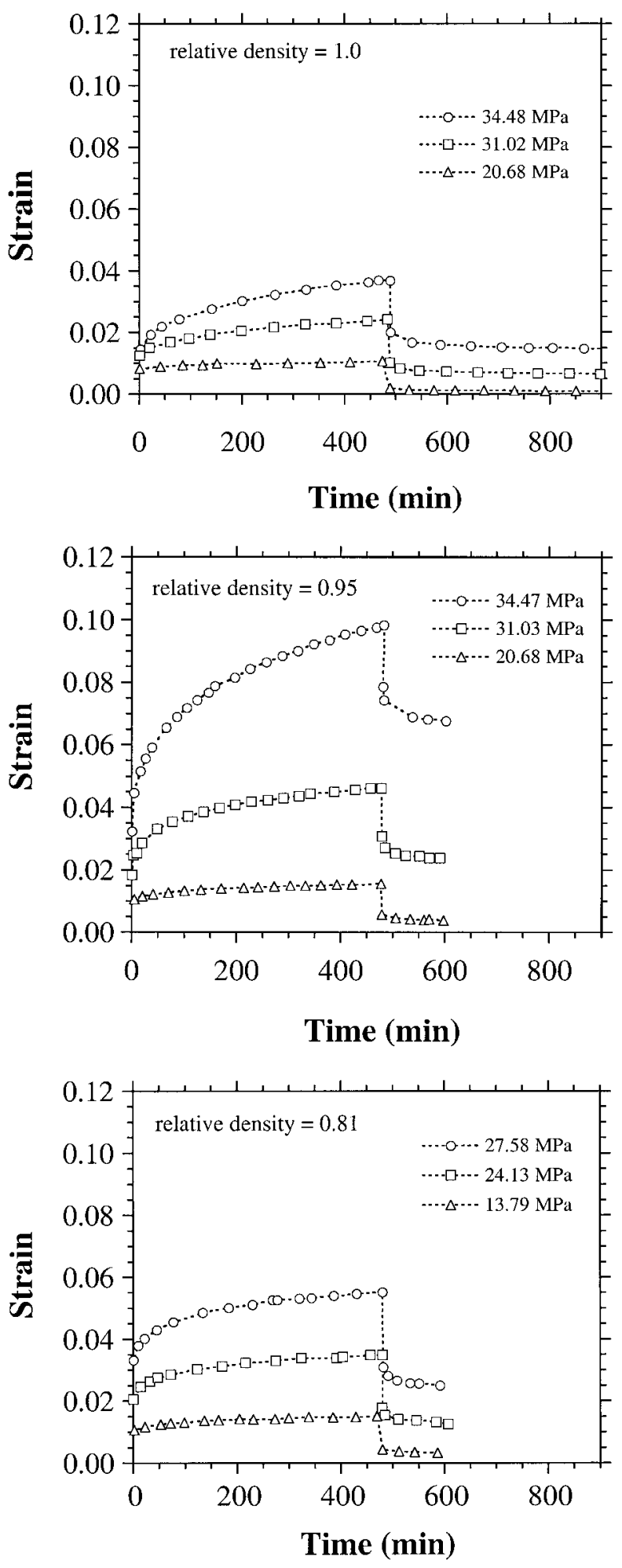

Figure 1. Experimental creep and recovery measurements: (a) relative density equal to 1.0 ; (b) relative density equal to 0.95 ; and (c) relative density equal to 0.81 .

ery strain is a significantly less sensitive function of the value of the creep stress than is the creep strain. 


\section{THE STRESS-DEPENDENT DYNAMIC CREEP AND RECOVERY COMPLIANCES}

The present method will make use of fast Fourier transforms, ${ }^{8-12}$ therefore the first analysis task is to fit the creep data of Figure 1 with an appropriate set of continuous functions, which may be evaluated at a regular interval and provide a reliable extrapolation to some range of times greater than that of the creep experiments. The present calculations linearly fit a second order series of Chebyshev polynomials to the creep strain versus log time data for this purpose. A discrete creep strain frequency spectra for each of the individual creep tests may now be constructed by fast Fourier transforming a strain list with an initial element equal to zero and subsequent elements obtained from the creep strain time function. The present calculations set the first strain time sample equal to zero and then evaluate the fitted creep strain functions every $10 \mathrm{~min}$ to produce a regular time sample list. The experimental discrete strain frequency components may then be obtained by fast Fourier transformation. ${ }^{13}$

$$
\tilde{E}\left(\frac{n}{N T}\right)=\frac{1}{\sqrt{N}} \sum_{k=0}^{N-1} \varepsilon(k T) \exp \left[2 \pi i \frac{n k}{N}\right]
$$

where $N$ is the number of elements in the time sample list and $T$ is the time sampling interval. The same numerical procedure is used for the stress assuming an ideal creep stress step time sequence.

$$
\sigma(k T)=\left\{0, \sigma_{0}, \sigma_{0}, \ldots, \sigma_{0}\right\}
$$

The discrete complex creep compliance frequency spectra may then be obtained by division of strain frequency components with corresponding stress frequency components.

$$
\begin{aligned}
& \tilde{J} \\
& =\left\{\frac{\tilde{E}\left(\frac{0}{N T}\right)}{\tilde{S}\left(\frac{0}{N T}\right)}, \frac{\tilde{E}\left(\frac{1}{N T}\right)}{\tilde{S}\left(\frac{1}{N T}\right)}, \frac{\tilde{E}\left(\frac{2}{N T}\right)}{\tilde{S}\left(\frac{2}{N T}\right)}, \ldots, \frac{\tilde{E}\left(\frac{N-1}{N T}\right)}{\tilde{S}\left(\frac{N-1}{N T}\right)}\right\}
\end{aligned}
$$

A discrete recovery strain frequency spectra for each of the individual creep tests may now be constructed by fast Fourier transforming a regu- lar recovery strain list with an initial element equal to zero and subsequent elements obtained as the difference between the extrapolated creep curve and a smoothly fitted recovery curve, the present calculations using a second order series of Bessel $\mathrm{K}$ functions as the fitting basis. The same numerical procedure is used for the stress again assuming an ideal recovery stress step time sequence.

$$
\sigma(k T)=\left\{0, \sigma_{0}, \sigma_{0}, \ldots, \sigma_{0}\right\}
$$

The discrete complex recovery compliance frequency spectra may now be obtained by division of recovery strain frequency components with corresponding stress frequency components.

$$
\begin{aligned}
& \tilde{J} \\
& =\left\{\frac{\tilde{E}\left(\frac{0}{N T}\right)}{\tilde{S}\left(\frac{0}{N T}\right)}, \frac{\tilde{E}\left(\frac{1}{N T}\right)}{\tilde{S}\left(\frac{1}{N T}\right)}, \frac{\tilde{E}\left(\frac{2}{N T}\right)}{\tilde{S}\left(\frac{2}{N T}\right)}, \ldots, \frac{\tilde{E}\left(\frac{N-1}{N T}\right)}{\tilde{S}\left(\frac{N-1}{N T}\right)}\right\}
\end{aligned}
$$

\section{THE DEPENDENCE OF THE DISCRETE COMPLEX COMPLIANCE FREQUENCY SPECTRA ON RELATIVE DENSITY AND STRESS}

The real and imaginary parts of the discrete complex creep and recovery compliance frequency spectra depend on frequency, relative density, and stress. ${ }^{15}$ An examination of such dependencies provides insight into the differences in creep and recovery behaviors and shows conditions over which the behavior of the material is relatively stable.

Figure 2 shows the real and imaginary components of the discrete complex creep and recovery compliance spectra for the relative density equal to 1.0 and 0.95 materials as functions of stress and frequency. As expected, higher stresses result in larger compliances for both materials. The compliances of the relative density equal to 0.95 case are significantly greater than that of the relative density equal to 1.0 case. Interestingly, the relative density and stress sensitivities of the complex creep compliances are much higher than that of the complex recovery compliances.

Figure 2 shows that the real and imaginary parts of the discrete complex creep and recovery compliances of microcellular polycarbonate ex- 

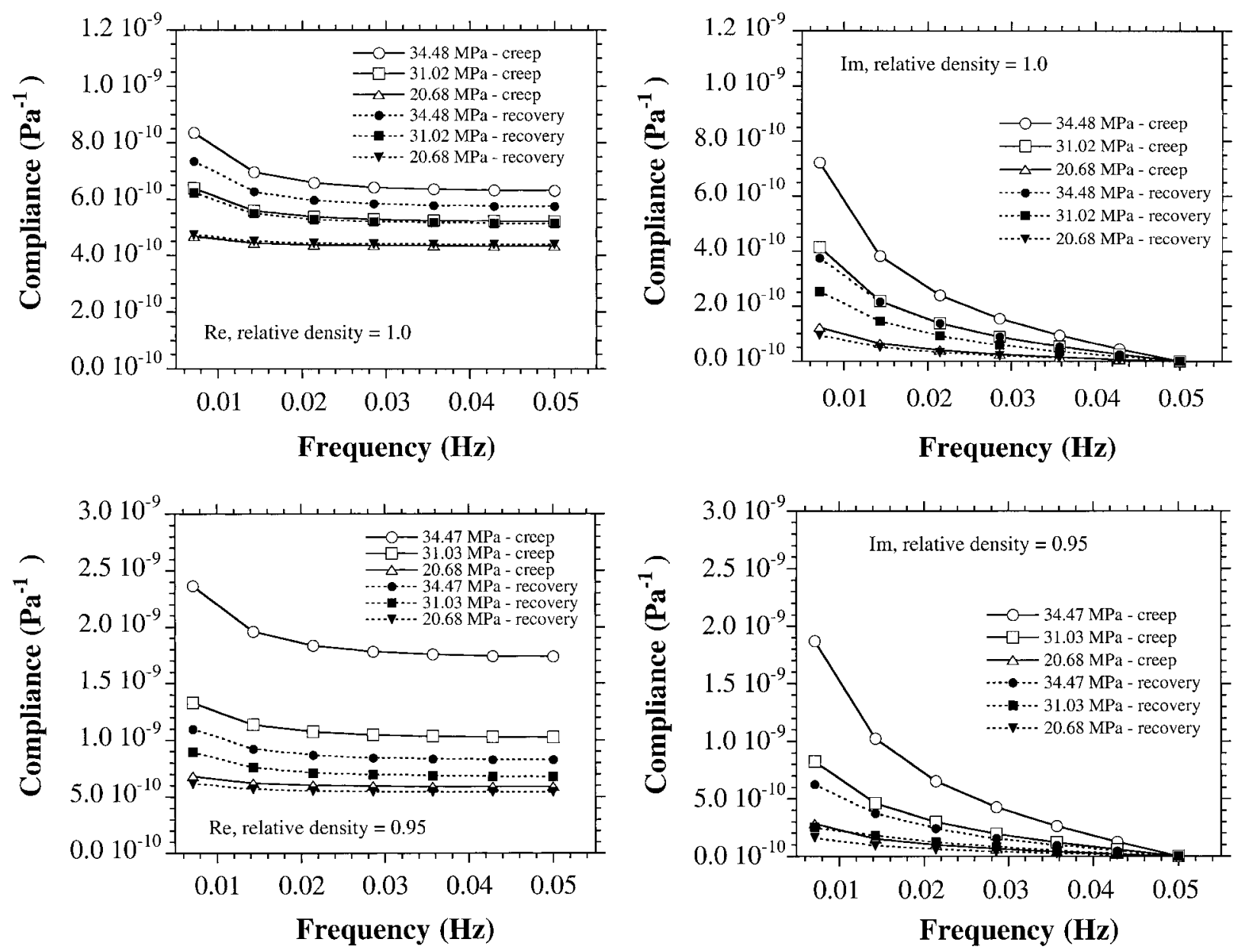

Figure 2. Components of the discrete complex creep and recovery compliance spectra for the relative density equal to 1.0 and 0.95 materials as functions of stress and frequency: (a) real component, relative density equal to 1.0; (b) imaginary component, relative density equal to 1.0 ; (c) real component, relative density equal to 0.95 ; (d) imaginary component, relative density equal to 0.95 .

hibit a smooth dependence on creep stress. Therefore, discrete stress-dependent complex compliance spectra may be constructed by assembling the fitting functions of the real and imaginary parts of each frequency component. Figure 3 shows the stress dependencies of the real (Re) and imaginary (Im) parts of the $0.00714 \mathrm{~Hz}$ component of the discrete complex creep and recovery compliances for microcellular polycarbonate with relative densities of $1.0,0.95$, and 0.81 . The figure shows that the magnitudes of the real and imaginary components of the compliance spectra all sharply increase with increasing stress. The figure further shows that the creep compliance spectra are much more sensitive to stress and relative density than is the case for the recovery compliance spectra.
Figure 4 shows the relative density dependence of the real part of the $0.00714 \mathrm{~Hz}$ component of the discrete complex creep compliance for microcellular polycarbonate at the experimental relative densities of $1.0,0.95$, and 0.81 as the applied stress varies from 22 to $34 \mathrm{MPa}$. The real part of the frequency component is linearly dependent on relative density under low applied stress. However, as the applied stress increases the real part of the frequency component becomes strongly nonlinearly dependent on relative density. Qualitatively similar behavior was exhibited by all of the other frequency components. This behavior clearly is the result of the interaction of the stress concentrating effects of the microcellular microstructure with the inherent nonlinear viscoelastic response of polycarbonate polymers. 

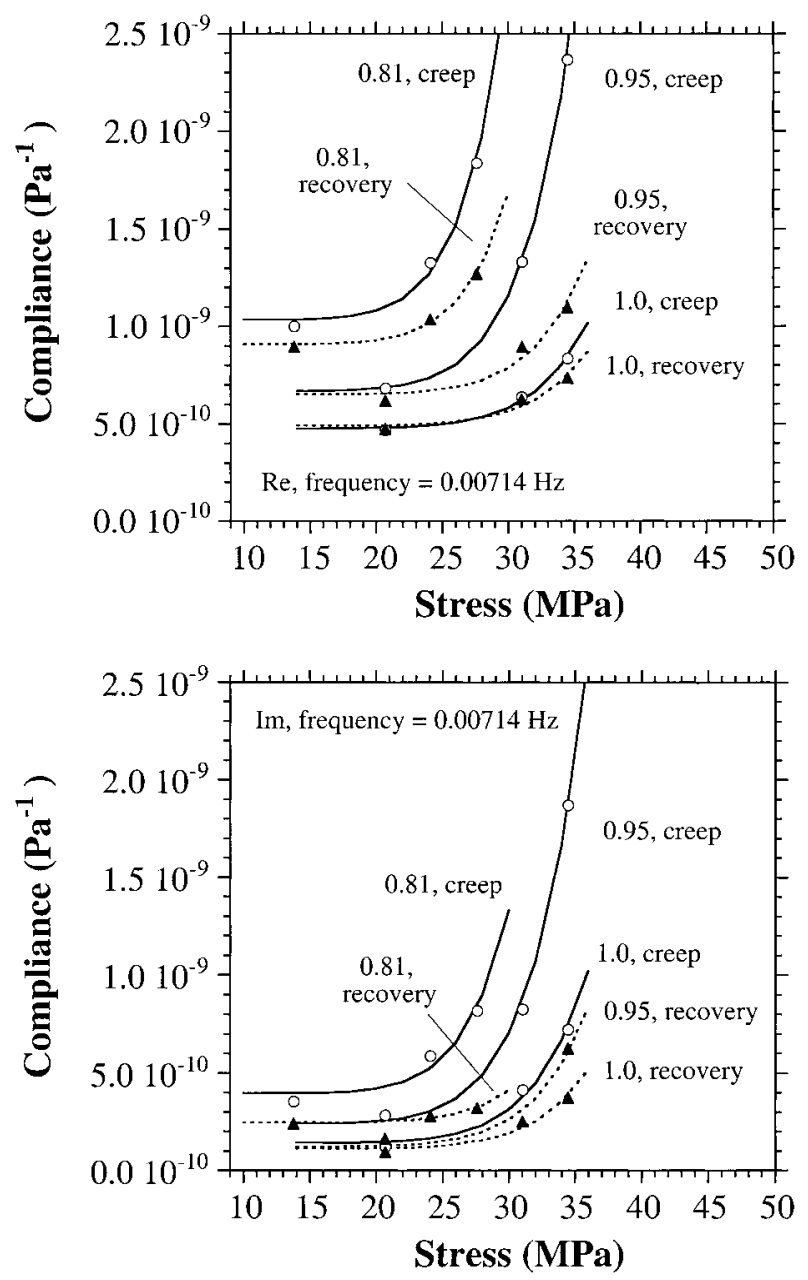

Figure 3. The stress dependencies of the real $(\mathrm{Re})$ and imaginary (Im) parts of the $0.00714 \mathrm{~Hz}$ component of the discrete complex creep and recovery compliances for microcellular polycarbonate with relative densities of $1.00,0.95$, and 0.81 . Points correspond to compliance spectra components obtained from experimental creep and recovery curves.

\section{COMPARISONS WITH EXPERIMENT}

The calculation of the creep process trajectory begins with the evaluation of the stress-dependent complex creep compliance spectra for a particular level of constant creep stress. The discrete creep strain frequency spectrum is then obtained by multiplying discrete complex creep compliance frequency components by corresponding discrete complex stress frequency components. The creep process time trajectory is then obtained by discrete inverse Fourier transformation.

$\varepsilon^{\text {creep }}(k t)$

$$
=\frac{1}{\sqrt{N}} \sum_{n=0}^{N-1} \tilde{J}^{c}\left(\frac{n}{N T}\right) \tilde{S}^{c}\left(\frac{n}{N T}\right) \exp \left[-2 \pi i \frac{n}{N}\right]
$$

The creep curve may now be calculated incrementally by summing the creep strain trajectory, resulting from the application of a constant creep stress at the start of each time increment, with the negative of the creep strain trajectory, resulting from the application of a constant creep stress at the end of each time increment for the current and all prior time increments in the manner of Pipkin and Rodgers. ${ }^{14}$

$$
\begin{aligned}
\varepsilon(k d t)=\sum_{i=1}^{k} \varepsilon_{i}^{\text {creep }}\left((k+1) d t, \sigma_{i}\right) & \\
& -\varepsilon_{i}^{\text {creep }}\left((k-i) d t, \sigma_{i}\right)
\end{aligned}
$$

where $\varepsilon^{\text {creep }}\left(t, \sigma_{i}\right)$ is the creep strain trajectory resulting from the constant creep stress $\sigma_{i}$.

The calculation of a recovery process history trajectory proceeds similarly, beginning with the evaluation of the stress-dependent complex recovery compliance spectra for a particular level of unload stress. The discrete recovery strain frequency spectrum may then be obtained by multiplying discrete complex recovery compliance frequency components by corresponding discrete complex unload stress frequency components. The recovery process time trajectory is then obtained by discrete inverse Fourier transformation.

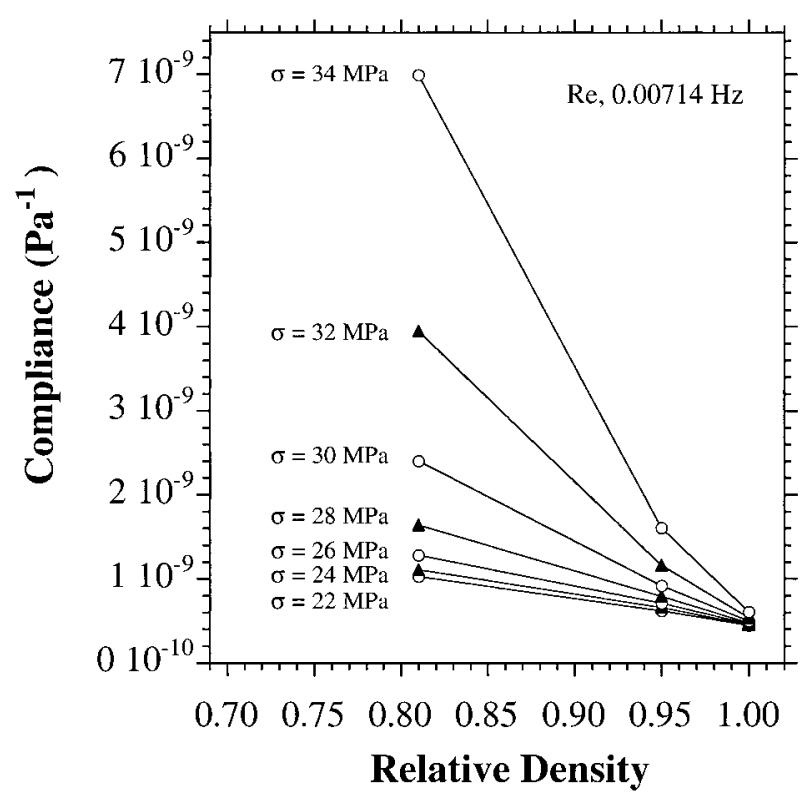

Figure 4. The relative density dependence of the real (Re) part of the $0.00714 \mathrm{~Hz}$ component of the discrete complex creep compliance for microcellular polycarbonate at the experimental relative densities of 1.0, 0.95, and 0.81 . 


$$
\begin{aligned}
& \varepsilon^{\text {recovery }}(k t) \\
& =\frac{1}{\sqrt{N}} \sum_{n=0}^{N-1} \tilde{J}^{r}\left(\frac{n}{N T}\right) \tilde{S}^{r}\left(\frac{n}{N T}\right) \exp \left[-2 \pi i \frac{n}{N}\right]
\end{aligned}
$$

The creep recovery curve may now be calculated incrementally by summing four individual strain trajectories: (a) the creep strain trajectory resulting from the application of a constant creep stress at the start of each time increment for the current and all prior time increments; (b) the negative of the creep strain trajectory resulting from the application of a constant creep stress at the end of each time increment for the current and all prior time increments; (c) the recovery strain trajectory resulting from the application of the constant recovery stress at the start of each time increment for the current and all prior time increments beyond the time of the release of the creep stress; and (d) the negative of the recovery strain trajectory resulting from the application of the constant recovery stress at the end of each time increment for the current and all prior time increments beyond the time of the release of the creep stress.

$$
\begin{aligned}
\varepsilon(k d t)= & \sum_{i=1}^{k} \varepsilon_{i}^{\text {creep }}\left((k+1-i) d t, \sigma_{i}\right) \\
& -\varepsilon_{i}^{\text {creep }}\left((k-i) d t, \sigma_{i}\right) \\
& +\sum_{i=1}^{k-j} \varepsilon_{i}^{\text {recovery }}\left((k-j+1-i) d t, \sigma_{i}\right) \\
& \quad-\varepsilon_{i}^{\text {recovery }}\left((k-j-i) d t, \sigma_{i}\right)
\end{aligned}
$$

where $\varepsilon^{\text {recovery }}{ }_{i}\left(t, \sigma_{i}\right)$ is the recovery strain trajectory resulting from the release of the constant creep stress $\sigma_{i}$, and $j$ is the number of time increments occurring before unloading.

Figure 5 compares experimental creep and recovery measurements with model calculations for each of the material types. The present calculations are specific to each relative density material type, however, the models may be evaluated at any reasonable stress. The figure shows that each of the models exhibits excellent quantitative agreement at all experimental stress levels, which is a simple result of the tight fit between the real and imaginary dynamic compliance stress functions with the experimentally based spectra at each of the applied stresses for each frequency component. Further experiments at new stress values could be easily included, and would result in better identification of the stress dependencies of each complex compliance frequency component.

\section{SUMMARY AND CONCLUSIONS}

This work reports a discrete stress-dependent complex creep and recovery compliance spectra method, which may be used to describe the mechanical response of nonlinear viscoelastic polymers. This work supports the following specific conclusions.

1. The real and complex parts of the discrete dynamic compliance obtained from creep measurements are smooth, easily fit functions of stress. Therefore, discrete complex compliance spectra with a continuous stress dependence may be constructed by assembling the real and imaginary fitting functions of each dynamic compliance frequency component.

2. The magnitudes of the real and imaginary components of the frequency components of the creep and recovery compliance spectra all sharply increase with increasing stress. The creep compliance components are much more sensitive to stress and relative density than is the case for the recovery compliance components.

3. The real part of each frequency component is linearly dependent on relative density under low applied stress. However, as the applied stress increases the real part of the frequency component becomes strongly nonlinearly dependent on relative density. This behavior is clearly the result of the interaction of the stress concentrating effects of the microcellular microstructure with the inherent nonlinear viscoelastic response of polycarbonate polymers.

4. The present calculations are specific to each relative density material type, however, the model may be evaluated at any reasonable stress. The model exhibits excellent quantitative agreement with the basis creep and recovery measurements at all experimental stress levels for each of the experimental relative density types, this is the result of the tight fit between the real and imaginary dynamic compliance stress functions with the experimentally based spectra at each of the applied stresses for each frequency component. 

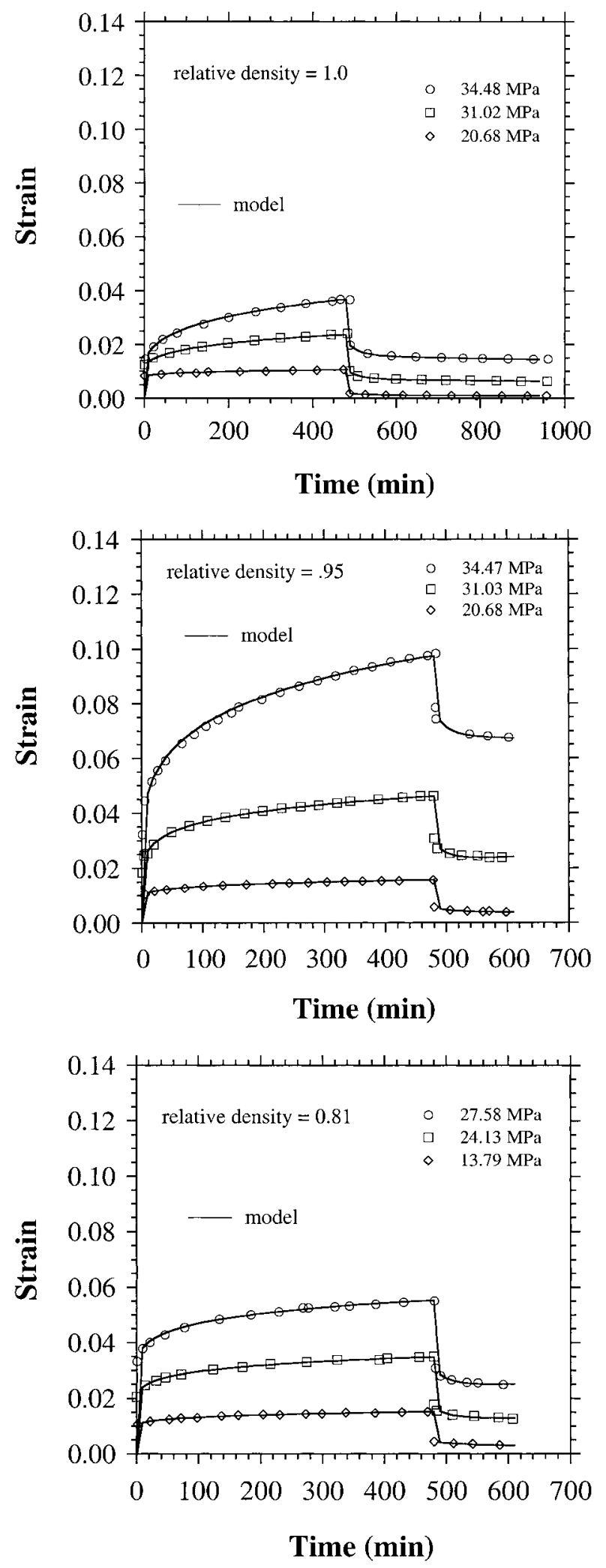

Figure 5. Comparison between short-duration experimental creep and recovery measurements and model calculations. The present calculations are specific to each relative density, however, the models may be evaluated at any reasonable stress.
The support of the Department of the Navy, Office of Naval Research under contract N00014-99-1-0276 and the direction of Program Officer Jan Lindberg is gratefully acknowledged; the content of this article does not necessarily reflect the position or the policy of the government, and no official endorsement should be inferred.

\section{REFERENCES AND NOTES}

1. Kumar, V. Microcellular Polymers: Novel Materials for the 21st Century. Prog Rubber Plast Technol 1993, 9(1), 54-70.

2. Kumar, V.; Weller, J. E. Production of Microcellular Polycarbonate Using Carbon Dioxide for Bubble Nucleation. ASME J Eng Indus 1994, 116, 413-420.

3. Kumar, V.; VanderWel, M.; Weller, J. E.; Seeler, K. A. Characterization of Tensile Behavior of Microcellular Polycarbonate. ASME J Eng Mater Technol 1994, 116, 39-445.

4. Kumar, V.; Weller, J. E. A Model for the Unfoamed Skin on Microcellular Foams. Polym Eng Sci 1994, 34, 169-173.

5. Barlow, C. M.S. Thesis, Department of Materials Science and Engineering, University of Washington, 1997.

6. Wing, G.; Pasricha, A.; Tuttle, M.; Kumar, V. Time Dependent Response of Polycarbonate and Microcellular Polycarbonate. Polym Eng Sci 1995, 35(8), 673-679.

7. Wing, G.; M.S. Thesis, Department of Mechanical Engineering, University of Washington, 1993.

8. Aspden, R. M. Aliasing Effects in Fourier Transforms of Monotonically Decaying Functions and the Calculation of Viscoelastic Moduli by Combining Transforms over Different Time Periods. J Phys D: Appl Phys 1991, 24, 803-808.

9. Holly, E. E.; Venkataraman, S. K.; Chambon, F.; Winter, H. H. Fourier Transform Mechanical Spectroscopy of Visoelastic Materials with Transient Structure. J Non-Newtonian Fluid Mech 1988, 27, 17-26.

10. Holmes, A. D.; Jing, F. The Experimental Nature of Stress Relaxation and Recovery of High-Frequency Information. J Phys D: Appl Phys 1994, 27, 2475-2479.

11. Kamath, V. M.; Mackely, M. R. Determination of Polymer Relaxation Moduli and Memory Functions Using Integral Transforms. J Non-Newtonian Fluid Mech 1989, 32, 119-144.

12. Arridge, R.G. C.; Barham, P. J. Fourier Transform Mechanical Spectroscopy. J Phys D: Appl Phys 1986, 19, L89-L96.

13. Brigham, E. O. The Fast Fourier Transform; Prentice Hall: Englewood Cliffs, New Jersey, 1974.

14. Pipkin, A. C.; Rogers, T. G. A Non-Linear Integral Representation for Viscoelastic Behavior. J Mech Phys Solids 1968, 16, 59-72.

15. Armstrong, W. D. A Stress Dependent Dynamic Compliance Spectra Approach to the Nonlinear Viscoelastic Response of Polymers. J Polym Sci Part B: Polym Phys 1998, 36, 2301-2309. 